\title{
The contribution of airway smooth muscle to airway narrowing and airway hyperresponsiveness in disease
}

\author{
J.G. Martin, A. Duguet, D.H. Eidelman
}

\begin{abstract}
The contribution of airway smooth muscle to airway narrowing and airway hyperresponsiveness in disease. J.G. Martin, A. Duguet, D.H. Eidelman. (C)ERS Journals Ltd 2000.

ABSTRACT: Airway hyperresponsiveness (AHR), the exaggerated response to constrictor agonists in asthmatic subjects, is incompletely understood. Changes in either the quantity or properties of airway smooth muscle (ASM) are possible explanations for AHR. Morphometric analyses demonstrate structural changes in asthmatic airways, including subepithelial fibrosis, gland hyperplasia/hypertrophy, neovascularization and an increase in ASM mass.

Mathematical modelling of airway narrowing suggests that, of all the changes in structure, the increase in ASM mass is the most probable cause of AHR. An increase in ASM mass in the large airways is more closely associated with a greater likelihood of dying from asthma than increases in ASM mass in other locations within the airway tree. ASM contraction is opposed by the elastic recoil of the lungs and airways, which appears to limit the degree of bronchoconstriction in vivo. The cyclical nature of tidal breathing applies stresses to the airway wall that enhance the bronchodilating influence of the lung tissues on the contracting ASM, in all probability by disrupting cross-bridges. However, the increase in ASM mass in asthma may overcome the limitation resulting from the impedances to ASM shortening imposed by the lung parenchyma and airway wall tissues. Additionally, ASM with the capacity to shorten rapidly may achieve shorter lengths and cause a greater degree of bronchoconstriction when stimulated to contract than slower ASM.

Changes in ASM properties are induced by the process of sensitization and allergen-exposure such as enhancement of phospholipase $\mathrm{C}$ activity and inositol phosphate turnover, and increases in myosin light chain kinase activity. Whether changes in ASM mass or biochemical/biomechanical properties form the basis for asthma remains to be determined.

Eur Respir J 2000; 16: 349-354.
\end{abstract}

Meakins-Christie Laboratories, Dept of Medicine, McGill University, Montreal, Quebec, Canada.

Correspondence: J.G. Martin

Meakins-Christie Laboratory

McGill University

3626 St Urbain Street

Montreal

Quebec

H2X 2P2 Canada

Fax: 15143987483

Keywords: Airways responsiveness airway smooth muscle

asthma

mechanics

modelling

Received: April 212000

Accepted after revision April 272000
The three defining characteristics of asthma are airway hyperresponsiveness (AHR), airway inflammation and intermittent airway obstruction. In the past, each of these characteristics has been the focus of research attention by investigators looking for abnormalities that could potentially contribute to the expression of disease. Neural control of airway calibre, epithelial modulation of airway smooth muscle (ASM) responses, airway inflammation, microvascular leakage and structural changes in the airways have been among the areas extensively explored. Although all of these factors have a place as modulators of the extent of airway narrowing in response to a contractile stimulus, the primary effector of airway responsiveness seems likely to be the airway smooth muscle itself. Some of the evidence for this is reviewed here.

\section{Mechanical factors influencing airway narrowing}

Mechanical factors are of paramount importance in determining the magnitude of bronchoconstriction induced

Previous articles in this series: No. 1: S.J. Gunst, D.D. Tang. The contractile apparatus and mechanical properties of airway smooth muscle. Eur Respir J 2000; 15: 600-616. No. 2: A. James, N. Carrol. Airway smooth muscle in health and disease; methods of measurement and relation to function. Eur Respir J 2000; 15: 782-789. No. 3: K.F. Chung. Airway smooth muscle cells: contributing to and regulating airway mucosal inflammation? Eur Respir J 2000; 15: 961-969. No. 4: I.P. Hall. Second messengers, ion channels and pharmacology of airway smooth muscle. Eur Respir J 2000; 15: 1120-1127. No. 5: S.J. Hirst, T.R. Walker, E.R. Chilvers. Phenotypic diversity and molecular mechanisms of airway smooth muscle proliferation in asthma. Eur Respir J 2000; 16: 159-177. 
by contractile agonists. Although support for this notion rests on circumstantial evidence rather than direct proof, bronchoconstriction is a function of ASM shortening, and thus of the mechanical forces acting to limit such shortening. It seems very likely that airway responsiveness reflects the balance between forces contracting ASM and the impedance to shortening of that muscle. Excised airway smooth muscle has an exceptional capacity to shorten in vitro (by $80-90 \%$ of its initial length) when stimulated. Shortening of this degree would result in complete airway closure if it were to take place in vivo. As airway closure does not appear to occur to any great extent following supramaximal stimulation in normal subjects, potent factors must exist that limit the degree of airway narrowing in vivo [1].

The analysis of bronchial provocation test results has been particularly helpful in providing insights into the mechanisms by which bronchoconstriction is limited in vivo. Lung volume is an important determinant of the degree of bronchoconstriction that can be induced by supramaximal doses of inhaled methacholine in normal subjects. Breathing at low lung volumes accentuates the degree of methacholine-induced bronchoconstriction markedly, whereas breathing at volumes greater than the functional residual capacity reduces the degree of bronchoconstriction [2]. The observation that breathing at low lung volumes greatly enhances the magnitude of airway narrowing whereas high volumes are "bronchoprotective" has been interpreted as evidence that the parenchymal attachments to the airway wall impose an impedance to airway narrowing. This impedance results from the elastic recoil of the parenchyma as well as from the forces of interdependence between the airway wall and the parenchymal attachments. Simply stated, the elastic recoil of the lung parenchyma helps to keep the airways open by transmitting a pressure equivalent to pleural pressure through the parenchymal attachments to the outer aspect of the airway walls. Indeed, the protective effect of these forces is amplified as a function of the degree of bronchoconstriction [3]. There is no conclusive evidence of impairment of the elastic properties of the lungs in asthma but it is possible that the transmission of the elastic impedance to the ASM may be affected by adventitial oedema.

The airway wall itself is likely to resist compression by causing contraction of ASM. The constitutive properties of the airway wall that help to prevent airway closure are likely to vary as a function of airway generation; airway cartilage is one of the components that resists airway compression. It has been postulated that alterations in the cartilage in asthma, through the action of proteolytic enzymes released by infiltrating leukocytes, might allow the airways to narrow more easily [4]. The mucosal surface of the airway wall forms folds which may vary in number as a function of both airway wall dimensions and wall material properties [5]. Thickening or stiffening of a thin inner structural layer, such as may result from increased subepithelial collagen levels in asthmatic airways, would be predicted to reduce the number of mucosal folds and thereby render the airway more susceptible to closure [5]. The formation of mucosal folds may also lead to an alteration in surface forces in the region of these interstices, causing a reduction in pressures and favouring transudation of fluid and further retraction of the airways [6].

\section{The contribution of airway smooth muscle to airway narrowing}

If lung elastic recoil or the constitutive properties of the airway walls alters the degree of induced bronchoconstriction by changing impedances on ASM, then a corollary is that the force of contraction of ASM should also influence airway narrowing. Indeed, it seems entirely plausible that the asthmatic state is the result of an increase in the forcegenerating capacity of ASM through alterations in its biochemistry or through an increase in the quantity of ASM. There is considerable evidence that the mass of ASM is increased in asthma, as discussed by JAMES and CARROLL elsewhere in this series [7]. It is presumed that increased ASM mass is the result of enhanced muscle growth, probably mediated by the complex mix of growth factors and contractile agonists that are present in the airways in asthmatic subjects. Furthermore, the site of the increase in muscle mass may be of prognostic significance. Subjects who have died of asthma show not only more ASM overall but also have a particularly noteworthy increase in ASM in the large airways compared to subjects with nonfatal asthma [8]. Whether an excess of ASM in large airways is simply a marker of a more extensive remodelling or accounts for the death of these subjects has not been resolved. However, the mechanical consequences of large airway narrowing should more profoundly affect airflow and the work of breathing than peripheral airway narrowing. Given the very large number of airways and assuming independence of their behaviour, the probability of synchronous closure of many airways is very low. This is certainly true for peripheral airways; the simultaneous closure or profound constriction of a sufficiently large number of airways to cause catastrophic increases in respiratory impedance and ventilatory failure would seem less probable than the simultaneous narrowing of a small number of central airways. Although it is unclear whether the impedances opposing airway narrowing are similar in central and peripheral airways, the potential for cartilage to assist in the stabilization of the central airways makes it likely that airway collapsibility does indeed vary at different sites in the airway tree.

The cyclical nature of lung inflation caused by tidal breathing also seems to protect against the induction of excessive airway narrowing by bronchoconstrictor agonists. Periodic inflation of the lungs by means of deep breaths appears to serve a bronchodilator function. Preventing normal subjects from taking deep breaths during inhalation of bronchoconstrictor agonists mimics the exaggerated response of an asthmatic subject [9], supporting the notion of breathing movements as modifiers of the extent of induced airway narrowing. Interestingly, asthmatic subjects seem to be relatively insensitive to the bronchodilator effects of deep breaths. Current evidence points to an action of tidal breathing on ASM function as an explanation for the above phenomena. Consistent with findings in intact human subjects, the application of cyclical stresses to airway segments in vitro potently reduces active stress generation by contracting ASM $[10,11]$. As illustrated in figure 1 , the magnitude of stretch required to reduce the active force generated by maximally stimulated airways by $50 \%$ was only $2 \%$ of muscle length [10]. This perturbation is only half that imposed by normal tidal breathing. The reduction of active stress by stretching has been 


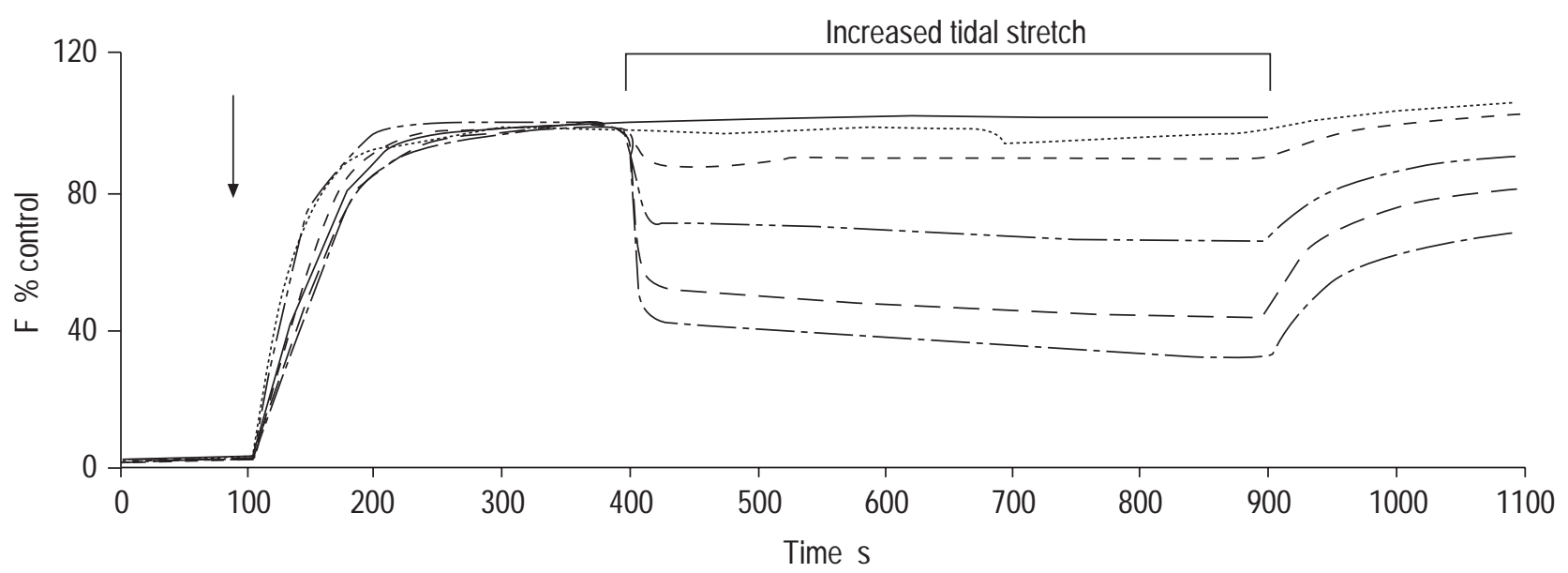

Fig. 1. - Time course of force $(\mathrm{F})$ in a tracheal smooth muscle strip. Each line corresponds to a different series of observations in the trachealis muscle at

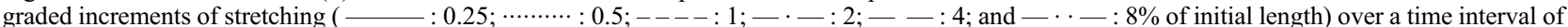
$400-900 \mathrm{~s}$. The vertical arrow denotes application of $10^{-4} \mathrm{M}$ acetylcholine. Note the profound reduction in force generation by the tidal stretching. (Adapted from [10].)

interpreted as resulting from the disruption of crossbridges in ASM cells, reducing the number of actin/myosin interactions and increasing their rate of turnover [12].

\section{Mathematical models of airway narrowing}

In an effort to understand the quantitative relationships between the many factors that potentially contribute to bronchial responsiveness, several investigators have turned to mathematical models $[13,14]$. These models examine the sensitivity of airway narrowing to structural changes such as ASM mass and distribution within the airway wall, airway wall thickening and lung volume. Each of these factors theoretically has the potential to increase airway responsiveness. Several of the earliest analyses of airway narrowing drew attention to the importance of the shortening capacity of ASM in airway responsiveness [1]. The sensitivity of airway luminal narrowing to airway wall thickness was addressed by Moreno et al. [15]. They demonstrated that, for any given degree of ASM shortening, the degree of encroachment on the airway lumen by the subepithelial tissues was a function of the thickness of these tissues. The potential for both of these properties of the airway to modulate the dose/response curve of the human lung to bronchoprovocation was clearly illustrated by modelling the airways as a simple tube. Subsequently, JAMES et al. [16] used a similar analysis to show that airway wall thickening of the degree found in asthma is of sufficient magnitude to substantially augment the degree of airway narrowing caused by any given degree of ASM shortening. Similarly, based on experimental data, it has been argued that, in asthma, peribronchial oedema also enhances airway responsiveness $[17,18]$, in large part by uncoupling the airway from the parenchyma. Finally, a consistent finding of all the modelling studies is that increases in ASM mass may lead to greater force generation within the airway wall, overcoming more easily the impedances opposing ASM shortening [13, 14].

An additional architectural feature of the airways that may have some bearing on airway responsiveness is the orientation of the ASM within the airway wall [19]. ASM is arranged in a spiral that may vary in its pitch [20].
Depending on the mechanical properties of the airway wall, in particular its resistance to compression in various axes, the orientation of the smooth muscle could markedly affect responsiveness. To date, there is insufficient experimental data to allow further interpretation of the predictions arising from the model.

MACKLEM [14] has recently performed a quantitative analysis of the various forces involved in airway narrowing (fig. 2). He computed the intrawall stress as a function of the degree of ASM shortening and estimated the effects of changing lung volume and ASM thickness. The analysis reveals several features of interest to the understanding

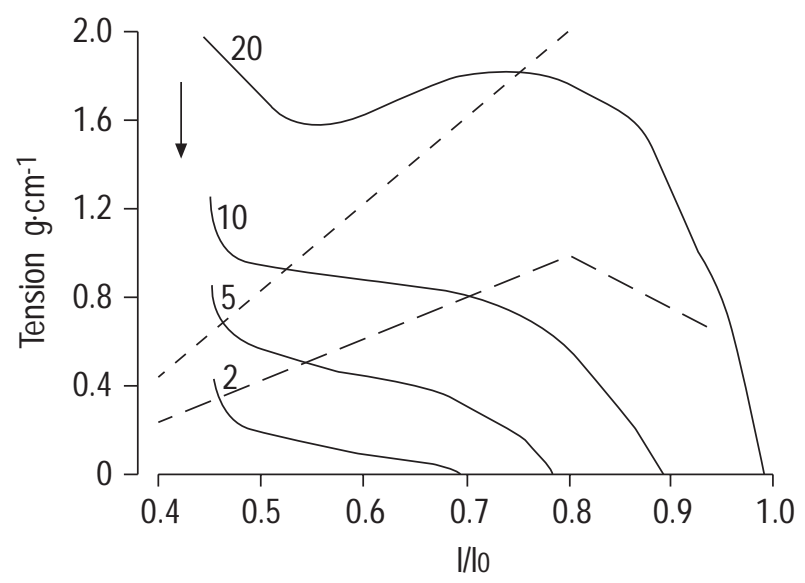

Fig. 2. - Tension developed by airway smooth muscle (ASM) in causing airway narrowing as a function of ASM length (1). The tension generation was calculated based on published data for canine airways. Assumptions have been made concerning the shear modulus of the parenchyma and the forces of interdependence between the airway and lung parenchyma. Each curve is based on calculations made at a given lung volume, as reflected in the magnitude of the transpulmonary pressure $(P \mathrm{tp})$. The figures labelling each curve represent initial $P$ tp in $\mathrm{cmH}_{2} \mathrm{O}$. At low $P$ tp, the degree of ASM shortening can be sufficient to cause airway closure (vertical arrow) without requiring tension generation by the muscle in excess of the maximum tension-generating capacity of the ASM. - _ ; - - - : length-tension curves). Note the dependence of the maximum tension available on the thickness of the ASM ( - - : : $\left.20 \mu \mathrm{m} ;-\frac{1}{2} 10 \mu \mathrm{m}\right) .10$ : ASM length at a $P$ tp of 30 $\mathrm{cmH}_{2} \mathrm{O}$. (Adapted from [14].) 
of airway narrowing. At low lung volumes (transpulmonary pressures), the stress development required to cause the airways to narrow is low and may result in airway closure before the maximal force-generating capacity of the muscle is reached. Increasing the transpulmonary pressure increases the stress requirements for airway narrowing into a zone that may be beyond the maximal capacity of the muscle to develop force, preventing airway closure. Conversely increasing the thickness of the muscle bundles may restore the contractile force to the levels necessary to cause airway closure despite the impedance. The modelling clearly illustrates the dependence of airway narrowing on the force of contraction of the ASM and the impedances to ASM shortening while also providing explanations for the sensitivity of bronchoconstriction to lung volume and ASM mass.

Some perspective on the relative importance of the various above-mentioned factors to airway responsiveness has been obtained by testing the effects of the observed structural changes in the airways of asthmatic subjects on the predicted dose/response curves to methacholine challenge. The weight of evidence suggests ASM thickness as the most likely modifier of the severity of acute asthma. For example, the modelling experiments of LAMBERT et al. [13] support the prediction that increased ASM mass is a more important cause of airway narrowing and hyperresponsiveness in asthma than is airway wall thickening. Indeed, the measured increases in ASM mass in asthma could entirely account for the substantial differences in methacholine dose/response curves between asthmatic and normal subjects. Although analysis of the importance of remodelling has focused mainly on airway submucosal thickening, alterations in the adventitial area have received less attention. Thickening of the adventitia could uncouple the airway from the parenchyma and exert a much more profound effect on airway responsiveness than changes in the airway wall interior to the ASM [14].

The above models treat airway narrowing as a quasistatic phenomenon. The observation that the application of cyclical stresses to ASM profoundly influences airway narrowing has raised important questions regarding the control of force generation by ASM. FREDBERG et al. [12] have developed the concept of airway narrowing as dynamically determined, such that the degree of airway narrowing depends on the relative rates of actin/myosin cross-bridge cycling and tidal stretching of ASM. This concept depends on the observation that, during contraction, smooth muscle appears to exhibit two phases. During the initial phase, corresponding to rapid cycling of actin/myosin cross-bridges, smooth muscle shortens rapidly while developing relatively little tension. During the subsequent "latchbridge" phase, the rate of cross-bridge cycling is greatly reduced, shortening velocity is slowed and tension development greatly increased [21]. By virtue of greater tension development under latch conditions, smooth muscle can resist the modulating influence of length changes. Applying these observations to the airways, FREDBERG and coworkers [12, 22] have postulated that hyperresponsiveness could result from resistance of ASM to the modulating influence of tidal breathing stretches of the airway wall on ASM. Specifically, they have proposed that, by shortening more quickly, ASM could enter the latch state before tidal stretch can exert its re- laxant effect. This hypothesis remains controversial, however, and more study is required to confirm that the shortening velocity of asthmatic ASM is indeed increased.

In considering the processes contributing to the degree of bronchoconstriction induced by bronchoconstrictive agonists, the potential importance of heterogeneity in airway narrowing across the airway tree should not be lost sight of. This question has been addressed in two recent studies investigating the contribution of heterogeneity to the increase in pulmonary impedance in response to bronchoconstrictive agonists [23, 24]. In both instances, the authors used the Horsfield asymmetric airway tree and computational models to determine lung resistance and elastance. These authors examined the effect of assigning differing degrees of variation (SDS) in individual airway diameters while maintaining the same mean airway diameter. Although different approaches were used, in both studies, the variability in airway narrowing itself accounted for additional increases in the calculated lung resistance. Presumably this reflects the bias introduced by the dependence of resistance on the radius of the airway to the fourth power, such that narrowed airways exert undue influence on overall lung resistance.

\section{Changes in the properties of airway smooth muscle in asthma}

The limit of ASM shortening may not be entirely a function of ASM mass and mechanical impedances to ASM contraction. Alterations in ASM biochemical properties may also be of considerable importance. There are changes in the function of ASM from the standpoint of its contractile apparatus $[25,26]$ and signalling pathways [27] as well as lesser-known synthetic capabilities. It has been argued that functional differences in ASM are present in asthmatic subjects because enhanced force generation by excised muscle does not seem to be entirely accounted for by increases in mass [28]. A number of experimental studies have provided convincing evidence of changes in the biochemical characteristics of ASM as a result of either sensitization or challenge with allergen. Tracheal and bronchial smooth muscle from sensitized dogs shows an increase in its maximal velocity of shortening [29], which appears to be attributable to an increase in the activity of myosin light chain kinase [26]. There was no increase in isometric force, however. The significance of changes in the velocity of contraction were not immediately apparent because preceding analyses of airway narrowing treated the airways in the bronchoconstricted state as being in static equilibrium. Two recent studies have demonstrated a relationship between airway responsiveness in vivo and the velocity of contraction of ASM within explanted lung tissues from the rat [30] and mouse [31]. The significance of the altered dynamic properties for AHR has been greatly clarified by the recent analyses of FREDBERG and coworkers [12, 22]. Perhaps asthmatic smooth muscle is, for some reason, resistant to the perturbing effects of the forces applied to it by the movements of the parenchyma. Alternatively, as mentioned above, the architectural changes in the airway wall, particularly thickening or oedema exterior to the 
ASM layer in the adventitial region of the wall may interfere with the transmission of forces from the parenchyma to ASM.

Activation of the contractile apparatus of ASM appears to be dependent on ASM length. For example, myosin light chain phosphorylation, a measure of the activation of the contractile apparatus, following stimulation of bovine ASM varies as a function of muscle length [32, 33], such that phosphorylation diminishes as muscle length is reduced. The precise explanation for this phenomenon has not been elucidated but a reduction in inositol phosphate turnover has been shown [34], suggesting that the degree of activation of phospholipase C (PLC) may be affected. An alternative possibility is that the availability of the membrane phospholipid, phosphatidylinositol 4,5- biphosphate, the substrate of PLC, may have been reduced. The observation that normal ASM responds to changes in length by altering the degree of myosin light chain phosphorylation raises the hypothetical possibility that, in asthma, ASM may be less sensitive to the inactivating effects of shortening.

Other alterations in the properties of ASM that have been shown to be caused by allergic sensitization or challenge are changes in membrane potential, PLC activity [27] and cyclic adenosine monophosphate synthesis in response to $\beta$-agonist stimulation [35]. In addition to the effects of allergic sensitization on the contractile apparatus of ASM, it is likely that there are also alterations in its synthesis of a variety of factors involved in the promotion of cell growth, matrix protein turnover and leukocyte adhesion $[35,36]$. ASM shows considerable plasticity of its phenotype in vitro [37] and it is probable that some of this plasticity may also be present in the airways in the setting of inflammation. Indeed, the fact that ASM can be stimulated to undergo a hyperplastic response on exposure of an experimental animal to allergen indicates that ASM can undergo substantial changes in phenotype in situ. Although ASM can change from an extreme contractile phenotype to a completely noncontractile secretory phenotype, there are, presumably, a range of phenotypic characteristics that may be expressed depending on the balance of cytokines, growth factors, contractile agonists and matrix proteins. Both the secretory and contractile phenotypes have the potential to influence the severity of asthma, by promoting airway remodelling or bronchospasm, respectively. ASM has the capacity to synthesize and release pro-inflammatory molecules such as eotaxin and regulated on activation, normal T-cell expressed and secreted (reviewed elsewhere in this series by CHUNG [38]), both noted for their eosinophil chemoattractant properties, although formal proof that ASM is involved in the promotion of inflammation is lacking.

\section{Animal models of airway remodelling in asthma}

The development of animal models of airway remodelling in asthma has lagged behind other attempts at recreating the features of asthma through the stimulus of allergen and other agents of potential relevance to asthma. However, there are now several reports indicating that certain airway structural changes can be induced in experimental animals. Allergen-challenged cats reportedly develop histopathological airway changes (eosinophil-rich inflammation, ASM growth and mucous gland enlarge- ment) that mimic very well the changes described for human asthmatic airways [39]. There is accompanying AHR. This is perhaps not very surprising since feline asthma is a condition that occurs spontaneously and which appears to be closely related to human asthma. However, it has also been possible to cause ASM hyperplasia in Brown Norway rats following allergic sensitization and repeated allergen exposure [40, 41]. A critical role for cysteinyl-leukotrienes in ASM growth and AHR has been confirmed [40, 42]. Guinea-pigs undergoing repeated allergen challenges have also been shown to incorporate bromodeoxyuridine (a thymidine analogue) into ASM [43], indicating that the cells enter the synthetic (S) phase of the cell cycle. Curiously no increase in ASM mass was detected, suggesting failure to progress through the cell cycle or accelerated cell death (apoptosis). A murine model of subepithelial fibrosis, a lesion that receives much attention but is of uncertain significance to the issue of airway narrowing in asthma, has also been reported [44]. These models will be very helpful in the dissection of the mechanisms of airway remodelling, but establishing cause and effect relationships with AHR may prove to be very problematic.

\section{Conclusions}

Experimental data and mathematical models suggest that alterations in the mass of airway smooth muscle or changes in its contractile properties may explain the excessive airway narrowing in asthma. However, the interaction between the contracting smooth muscle and the surrounding structures, including the constituents of the airway wall and lung parenchyma, may be of equal importance. In particular, the cyclical stresses applied to airway smooth muscle by tidal breathing or episodic deep breaths may serve an important function in maintaining the airways in a dilated state. Several biochemical changes of potential importance to airway hyperresponsiveness, including increased activity of myosin light chain kinase and phospholipase $\mathrm{C}$, have been shown to result from allergic sensitization. Perhaps alterations in the dynamic properties of airway smooth muscle, such as increases in the velocity of shortening, may permit the muscle to better resist the dilating influence of cyclical stress imposed by tidal breathing. Whether hyperplastic airway smooth muscle undergoes changes in its dynamic properties merits exploration.

\section{References}

1. Macklem PT. Bronchial hyperresponsiveness. Chest 1987; 91: 189S-191S.

2. Ding DJ, Martin JG, Macklem PT. Effects of lung volume on maximal methacholine induced bronchoconstriction in normal humans. J Appl Physiol 1987; 62: 1324-1330.

3. Sasaki H, Hoppin FG Jr, Takishima T. Peribronchial pressure in excised dog lungs. $J$ Appl Physiol 1978; 45: 858869.

4. Moreno RH, Lisboa C, Hogg JC, Pare PD. Limitation of airway smooth muscle shortening by cartilage stiffness and lung elastic recoil in rabbits. J Appl Physiol 1993; 75 : 738-744.

5. Wiggs BR, Hrousis CA, Drazen JM, Kamm RD. On the mechanism of mucosal folding in normal and asthmatic airways. J Appl Physiol 1997; 83: 1814-1821.

6. Yager D, Shore S, Drazen JM. Airway luminal liquid. 
Sources and role as an amplifier of bronchoconstriction. Am Rev Respir Dis 1991; 143: S52-S54.

7. James AL, Carroll N. Airway smooth muscle in health and disease; methods of measurement and relation to function. Eur Respir J 2000; 15: 782-789.

8. Carroll N, Elliot J, Morton A, James A. The structure of large and small airways in nonfatal and fatal asthma. Am Rev Respir Dis 1993; 147: 405-410.

9. Skloot G, Permutt S, Togias A. Airway hyperresponsiveness in asthma: a problem of limited smooth muscle relaxation with inspiration. J Clin Invest 1995; 96: 2393-2403.

10. Fredberg JJ, Inouye D, Miller B, et al. Airway smooth muscle, tidal stretches, and dynamically determined contractile states. Am J Respir Crit Care Med 1997; 156: $1752-1759$.

11. Gunst SJ, Stropp JQ. Pressure-volume and length-stress relationships in canine bronchi in vitro. $J$ Appl Physiol 1988; 64: 2522-2531.

12. Fredberg JJ, Inouye DS, Mijailovich SM, Butler JP. Perturbed equilibrium of myosin binding in airway smooth muscle and its implications in bronchospasm. Am J Respir Crit Care Med 1999; 159: 959-967.

13. Lambert RK, Wiggs BR, Kuwano K, Hogg JC, Pare PD. Functional significance of increased airway smooth muscle in asthma and COPD. J Appl Physiol 1993; 74: 27712781.

14. Macklem PT. A theoretical analysis of the effect of airway smooth muscle load on airway narrowing. Am J Respir Crit Care Med 1996; 153: 83-89.

15. Moreno RH, Hogg JC, Pare PD. Mechanics of airway narrowing. Am Rev Respir Dis 1986; 133: 1171-1180.

16. James AL, Pare PD, Hogg JC. The mechanics of airway narrowing in asthma. Am Rev Respir Dis 1989; 139: 242246.

17. Brown RH, Zerhouni EA, Mitzner W. Airway edema potentiates airway reactivity. J Appl Physiol 1995; 79: 1242-1248.

18. Brown RH, Mitzner W, Wagner EM. Interaction between airway edema and lung inflation on responsiveness of individual airways in vivo. J Appl Physiol 1997; 83: 366370.

19. Bates JH, Martin JG. A theoretical study of the effect of airway smooth muscle orientation on bronchoconstriction. J Appl Physiol 1990; 69: 995-1001.

20. Lei M, Ghezzo H, Chen MF, Eidelman DH. Airway smooth muscle orientation in intraparenchymal airways. $J$ Appl Physiol 1997; 82: 70-77.

21. Hai CM, Murphy RA. Cross-bridge phosphorylation and regulation of latch state in smooth muscle. Am J Physiol 1988; 254: C99-C106.

22. Solway J, Fredberg JJ. Perhaps airway smooth muscle dysfunction contributes to asthmatic bronchial hyperresponsiveness after all. Am J Respir Cell Mol Biol 1997; 17: 144-146.

23. Thorpe CW, Bates JH. Effect of stochastic heterogeneity on lung impedance during acute bronchoconstriction: a model analysis. J Appl Physiol 1997; 82: 1616-1625.

24. Gillis HL, Lutchen KR. Airway remodeling in asthma amplifies heterogeneities in smooth muscle shortening causing hyperresponsiveness. J Appl Physiol 1999; 86: 2001-2012.

25. Antonissen LA, Mitchell RW, Kroeger EA, Kepron W, Tse KS, Stephens NL. Mechanical alterations of airway smooth muscle in a canine asthmatic model. J Appl Physiol 1979; 46: 681-687.

26. Jiang H, Rao K, Halayko AJ, Liu X, Stephens NL. Ragweed sensitization-induced increase of myosin light chain kinase content in canine airway smooth muscle. Am J Respir Cell Mol Biol 1992; 7: 567-573.
27. Salari H, Yeung M, Howard S, Schellenberg RR. Increased contraction and inositol phosphate formation of tracheal smooth muscle from hyperresponsive guinea pigs. J Allergy Clin Immunol 1992; 90: 918-926.

28. Thomson RJ, Bramley AM, Schellenberg RR. Airway muscle stereology: implications for increased shortening in asthma. Am J Respir Crit Care Med 1996; 154: 749757.

29. Jiang H, Rao K, Halayko AJ, Kepron W, Stephens NL. Bronchial smooth muscle mechanics of a canine model of allergic airway hyperresponsiveness. J Appl Physiol 1992; 72: 39-45.

30. Tao FC, Tolloczko B, Eidelman DH, Martin JG. Enhanced $\mathrm{Ca}^{2+}$ mobilization in airway smooth muscle contributes to airway hyperresponsiveness in an inbred strain of rat. Am J Respir Crit Care Med 1999; 160: 446-453.

31. Duguet A, Biyah K, Minshall E, et al. Bronchial responsiveness among inbred mouse strains. Role of airway smooth-muscle shortening velocity. Am J Respir Crit Care Med 2000; 161: 839-848.

32. Yoo J, Ellis R, Morgan KG, Hai CM. Mechanosensitive modulation of myosin phosphorylation and phosphatidylinositol turnover in smooth muscle. Am J Physiol 1994; 267: C1657-C1665.

33. Mehta D, Wu MF, Gunst SJ. Role of contractile protein activation in the length-dependent modulation of tracheal smooth muscle force. Am J Physiol 1996; 270: C243C252.

34. An SS, Hai CM. Mechanical strain modulates maximal phosphatidylinositol turnover in airway smooth muscle. Am J Physiol 1999; 277: L968-L974.

35. Hakonarson H, Herrick DJ, Grunstein MM. Mechanism of impaired $\beta$-adrenoceptor responsiveness in atopic sensitized airway smooth muscle. Am J Physiol 1995; 269: L645-L652.

36. John M, Hirst SJ, Jose PJ, et al. Human airway smooth muscle cells express and release RANTES in response to $\mathrm{T}$ helper 1 cytokines: regulation by $\mathrm{T}$ helper 2 cytokines and corticosteroids. J Immunol 1997; 158: 1841-1847.

37. Halayko AJ, Camoretti-Mercado B, Forsythe SM, et al. Divergent differentiation paths in airway smooth muscle culture: induction of functionally contractile myocytes. Am J Physiol 1999; 276: L197-L206.

38. Chung KF. Airway smooth muscle cells: contributing to and regulating airway mucosal inflammation? Eur Respir $J$ 2000; 15: 961-969.

39. Padrid P, Snook S, Finucane T, et al. Persistent airway hyperresponsiveness and histologic alterations after chronic antigen challenge in cats. Am J Respir Crit Care Med 1995; 151: 184-193.

40. Salmon M, Walsh DA, Koto H, Barnes PJ, Chung KF. Repeated allergen exposure of sensitized Brown-Norway rats induces airway cell DNA synthesis and remodelling. Eur Respir J 1999; 14: 633-641.

41. Sapienza S, Du T, Eidelman DH, Wang NS, Martin JG. Structural changes in the airways of sensitized brown Norway rats after antigen challenge. Am Rev Respir Dis 1991; 144: 423-427.

42. Wang CG, Du T, Xu LJ, Martin JG. Role of leukotriene $\mathrm{D}_{4}$ in allergen-induced increases in airway smooth muscle in the rat. Am Rev Respir Dis 1993; 148: 413-417.

43. Wang ZL, Walker BA, Weir TD, et al. Effect of chronic antigen and $\beta_{2}$-agonist exposure on airway remodeling in guinea pigs. Am J Respir Crit Care Med 1995; 152: 2097-2104.

44. Blyth DI, Pedrick MS, Savage TJ, Hessel EM, Fattah D. Lung inflammation and epithelial changes in a murine model of atopic asthma. Am J Respir Cell Mol Biol 1996; 14: $425-438$. 